\title{
BMJ Open Prospective multicentre cohort trial on acute appendicitis and microbiota, aetiology and effects of antimicrobial treatment: study protocol for the MAPPAC (Microbiology APPendicitis ACuta) trial
}

\author{
Sanja Vanhatalo, ${ }^{1,2}$ Eveliina Munukka, ${ }^{3,4}$ Suvi Sippola, ${ }^{2,5}$ Sirpa Jalkanen, ${ }^{6}$ \\ Juha Grönroos, ${ }^{2,5}$ Harri Marttila, ${ }^{7}$ Erkki Eerola, ${ }^{1,4}$ Saija Hurme, ${ }^{8}$ Antti J Hakanen, ${ }^{1,4}$ \\ Paulina Salminen, ${ }^{2,5,9}$ The APPAC collaborative study group
}

To cite: Vanhatalo S,

Munukka E, Sippola S, et al. Prospective multicentre cohort trial on acute appendicitis and microbiota, aetiology and effects of antimicrobial treatment: study protocol for the MAPPAC (Microbiology APPendicitis ACuta) trial. BMJ Open 2019;9:e031137. doi:10.1136/ bmjopen-2019-031137

- Prepublication history and additional material for this paper are available online. To view these files, please visit the journal online (http://dx.doi. org/10.1136/bmjopen-2019031137).

Received 17 April 2019 Revised 19 July 2019 Accepted 02 August 2019

Check for updates

(c) Author(s) (or their employer(s)) 2019. Re-use permitted under CC BY-NC. No commercial re-use. See rights and permissions. Published by BMJ.

For numbered affiliations see end of article.

Correspondence to Dr Paulina Salminen; paulina.salminen@tyks.fi

\section{ABSTRACT}

Introduction Based on the epidemiological and clinical data, acute appendicitis can present either as uncomplicated or complicated. The aetiology of these different appendicitis forms remains unknown. Antibiotic therapy has been shown to be safe, efficient and cost-effective for CT-confirmed uncomplicated acute appendicitis. Despite appendicitis being one of the most common surgical emergencies, there are very few reports on appendicitis aetiology and pathophysiology focusing on the differences between uncomplicated and complicated appendicitis.

Microbiology APPendicitis ACuta (MAPPAC) trial aims to evaluate these microbiological and immunological aspects including immune response in the aetiology of these different forms also assessing both antibiotics non-responders and appendicitis recurrence. In addition, MAPPAC aims to determine antibiotic and placebo effects on gut microbiota composition and antimicrobial resistance.

Methods and analysis MAPPAC is a prospective clinical trial with both single-centre and multicentre arm conducted in close synergy with concurrent trials APPendicitis ACuta II (APPAC II) (per oral (p.o.) vs intravenous+p.o. antibiotics, NCT03236961) and APPAC III (double-blind trial placebo vs antibiotics, NCT03234296) randomised clinical trials. Based on the enrolment for these trials, patients with CT-confirmed uncomplicated acute appendicitis are recruited also to the MAPPAC study. In addition to these conservatively treated randomised patients with uncomplicated acute appendicitis, MAPPAC will recruit patients with uncomplicated and complicated appendicitis undergoing appendectomy. Rectal and appendiceal swabs, appendicolith, faecal and serum samples, appendiceal biopsies and clinical data are collected during the hospital stay for microbiological and immunological analyses in both study arms with the longitudinal study arm collecting faecal samples also during follow-up up to 12 months after appendicitis treatment.
Strengths and limitations of this study

- To our knowledge, Microbiology APPendicitis ACuta (MAPPAC) is the first prospective trial comparing the role of microbiology and immunology including immune response in the aetiology of uncomplicated and complicated acute appendicitis in a large patient cohort consisting of CT-diagnosed patients also specifically evaluating appendicoliths and recurrent appendicitis after initial successful conservative treatment.

- The strong synergy between two ongoing randomised clinical trials APPendicitis ACuta II and III enabling a large prospective patient cohort of acute appendicitis patients with associated clinical data to be assessed with the microbiological and immunological findings.

- The application of next-generation sequencing combined with traditional culturing methods will provide extensive information about the microbiological factors in the aetiology of complicated and uncomplicated acute appendicitis, also presenting a challenge in differentiating between aetiological and non-aetiological microbiota in the specimens.

- The comprehensive approach of the MAPPAC study acquiring a large set of samples in the emergency surgery setting presents a challenge to surgeons on call and some patients may not have all study samples available.

Ethics and dissemination This study has been approved by the Ethics Committee of the Hospital District of Southwest Finland (Turku University Hospital, approval number ATMK:142/1800/2016) and the Finnish Medicines Agency. Results of the trial will be published in peer-reviewed journals.

Trial registration number NCT03257423 


\section{INTRODUCTION}

Acute appendicitis is one of the most common causes of abdominal pain in the emergency departments. The lifetime risk of acute appendicitis in males is $8.6 \%$ and $6.7 \%$ in females ${ }^{1}$ with recent meta-analysis showing an increasing trend in appendicitis incidence in the industrialised countries. ${ }^{2}$ Based on the epidemiological and clinical data, acute appendicitis can present either as uncomplicated or complicated with the majority of cases being uncomplicated.

The different epidemiological trends of uncomplicated and complicated acute appendicitis indicate different pathophysiology. ${ }^{3}$ Despite the high incidence of acute appendicitis, there are very few reports on appendicitis aetiology and pathophysiology especially focusing on the possible differences between uncomplicated and complicated acute appendicitis. Complicated acute appendicitis in this trial is defined as a finding of perforation, appendicolith, abscess or a suspicion of tumour. Appendicolith is a calcified faecal concretion in the appendix and it is the most common form of complicated acute appendicitis. Even though the first thorough study on appendicoliths was already reported in 1966, ${ }^{4}$ the information about appendiceal calculi is scarce. Obstruction of the appendiceal lumen caused by an appendicolith, lymphoid hyperplasia or swelling has been evaluated to be the primary cause of appendicitis and bacterial overgrowth has been considered as a consequence. ${ }^{5}$ However, bacterial infection has also been proposed as the primary cause of appendicitis. ${ }^{67}$ Bacteroides species are reported to be one of most common bacterial findings in appendicitis. ${ }^{8}$ Further, certain members of the Fusobacteria, especially Fusobacterium nucleatum and Fusobacterium necrophorum, are present in most appendicitis samples. ${ }^{6}$ The most common aerobic bacteria organism detected by culturing is Escherichia coli, but also Klebsiella pneumoniae, Streptococcus spp, Enterococcus $s p p$ and Pseudomonas aeruginosa have been reported. ${ }^{1011}$ To our knowledge, only one study with a very small number of patients has characterised the adult appendiceal microbiota profile with next-generation sequencing (NGS) methods. Appendix seems to have diverse microbiota, including both commensal species from gut microbiota (GM) and opportunistic pathogens. ${ }^{12} 13$ Since the interindividual variability in the microbial composition of the appendix samples is high, ${ }^{12}$ a larger number of appendicitis patients are needed to draw the microbiological conclusions. Since most of the species identified from the appendix with both culturing and NGS methods can also be part of normal GM, it is challenging to determine their role in the infection. ${ }^{14}$ In addition, the immune response and predisposition for infection by specific bacteria vary between individuals. Consequently, innate immunity is considered to be a contributing factor in the development of complicated appendicitis. ${ }^{15}$ In conclusion, several factors have been proposed to take part in the development of appendicitis, most importantly, obstruction of the lumen and bacterial infection. Research on aetiopathology has largely been focusing on complicated acute appendicitis. $^{5-7911}$
Antimicrobials decrease GM diversity, richness and species variation, and cause a perturbation in its overall balance. Particularly broad-spectrum antibiotics have been shown to have a long-term effect on GM profile. ${ }^{16} 17$ A prolonged disturbance in GM and the following imbalance with the host and its immune system have been associated with a variety of diseases, such as inflammatory bowel disease $^{18}$ and type 2 diabetes. ${ }^{19}$ Antibiotic use can further lead to increased prevalence of antibiotic resistant bacteria and antibiotic resistance genes. ${ }^{20-22}$ Although the effects of antibiotic treatment on the development of antimicrobial resistance (AMR) are less clear in countries with lower prevalence of resistant bacteria, ${ }^{23} 24$ the evaluation of antibiotic treatment effects on GM is essential in the treatment of acute appendicitis.

Appendectomy has unquestionably been the standard treatment for acute appendicitis for over a century with more than 300000 appendectomies performed annually in the USA. ${ }^{3}$ The original APPendicitis ACuta (APPAC) trial reported that at long-term follow-up, the majority of patients with CT-confirmed uncomplicated acute appendicitis were successfully treated with antibiotics, and those patients who required later appendectomy did not have increased or major complications. ${ }^{25}$ Antibiotic therapy for CT-confirmed uncomplicated acute appendicitis has been shown to be safe, efficient and cost-effective both in adult and paediatric patients. ${ }^{27-31}$ APPAC II trial aims to optimise antibiotic treatment for CT-confirmed uncomplicated acute appendicitis in order to both shorten the hospital stay and restrict the antibacterial spectrum. The APPAC III trial aims to assess symptomatic treatment of uncomplicated acute appendicitis and the role of antibiotics in the resolution of uncomplicated appendicitis. The Microbiology APPendicitis ACuta (MAPPAC) trial patient enrolment is based on the ongoing APPAC II and III randomised multicentre clinical trials of our study group. To our knowledge, there are so far no similar large microbiological studies focusing on acute appendicitis performed in conjunction with large clinical trials with prospective access to both uncomplicated and complicated appendicitis patients. MAPPAC trial aims to evaluate the possible role and differences in the microbiological aetiology of complicated and uncomplicated appendicitis with a special reference to the presence of an appendicolith. In addition, MAPPAC aims to evaluate the immunological and microbiological factors involved in appendicitis recurrence after successful initial antibiotic therapy. In the longitudinal study arm, we also aim to assess the effects of antibiotic and placebo treatment on the GM profile and the effects of hospital stay duration on the AMR reservoir of the GM.

\section{METHODS AND ANALYSIS}

\section{Study design}

MAPPAC is a prospective clinical trial with a single-centre and a multicentre arm. The single-centre study arm at Turku University Hospital aims to determine the possible differences in the aetiology of complicated and uncomplicated 


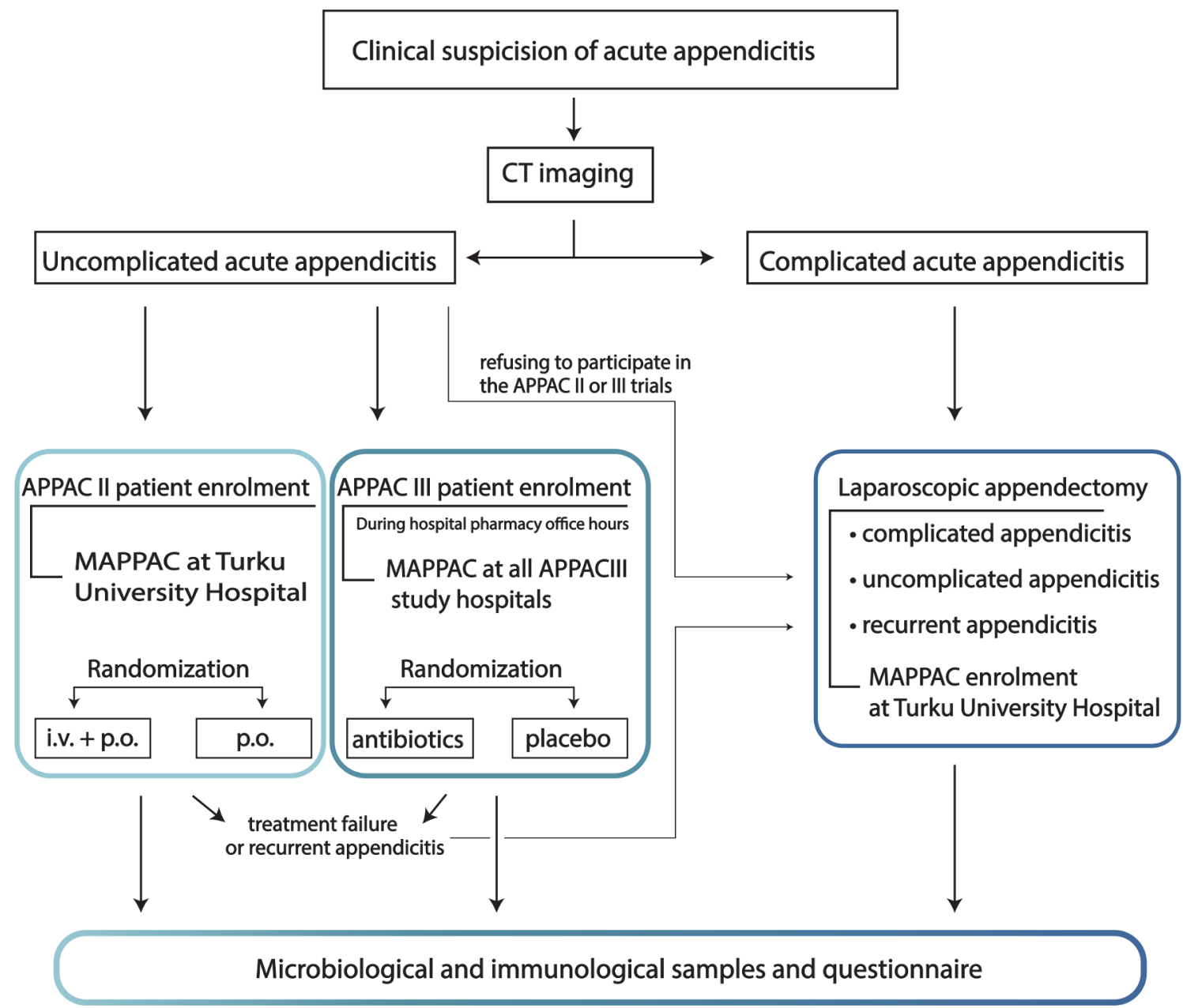

Figure 1 The synergy between MAPPAC, APPAC II and III studies. APPAC II, APPendicitis ACuta II; i.v, intravenous; MAPPAC, Microbiology APPendicitis ACuta; p.o., per oral.

acute appendicitis with a special reference to the role and aetiology of appendicolith. In the multicentre longitudinal arm, MAPPAC trial concentrates on assessment of possible immunological and microbiological factors involved in initial non-responders or appendicitis recurrence after the successful initial antibiotic therapy at long-term follow-up. In this longitudinal multicentre study arm, we also aim to evaluate the effects of antibiotic and placebo treatment on the GM and the effects of the duration of the hospital stay on the possible AMR reservoir within the GM. MAPPAC protocol was drafted in accordance with the Standard Protocol Items: Recommendations for Interventional Trials statement. ${ }^{32}$

The three ongoing study entities (APPAC II, APPAC III and MAPPAC) are strongly interconnected having a common study aim and a patient enrolment population (figure 1). APPAC II trial is a randomised open-label, non-inferiority multicentre trial to compare intravenous antibiotic therapy followed by per oral (p.o.) antibiotics with p.o. monotherapy in the treatment of uncomplicated acute appendicitis (NCT03236961). ${ }^{33}$ APPAC III trial is a randomised double-blind, placebo-controlled, superiority multicentre study to compare antibiotic therapy with placebo in the treatment of uncomplicated acute appendicitis (NCT03234296).${ }^{34}$ All incoming patients are informed of all ongoing trials. All patients invited to participate in APPAC II and III trials will be invited to participate in the MAPPAC trial. Patients recruited for the APPAC II or III trial are asked to sign a separate consent form for the MAPPAC trial allowing for the use of their data and collection of microbiological samples. The study flow is illustrated in figure 2 .

\section{Patient selection}

Eligible for inclusion are all adult patients 18-60 years presenting with either a CT-confirmed uncomplicated or complicated appendicitis or patients with a suspected recurrent appendicitis after initial successful antibiotic therapy for uncomplicated acute appendicitis. MAPPAC single-centre arm will enrol patients with both uncomplicated (both APPAC II and III trials and patients declining to participate in these trials undergoing appendectomy) and complicated acute appendicitis (patients excluded from APPAC II and III trials) at Turku University Hospital. The enrolment of uncomplicated acute appendicitis patients participating in the APPAC III trials will be performed at all APPAC III hospitals (all five Finnish university hospitals: Turku, Helsinki, Tampere, Oulu and 


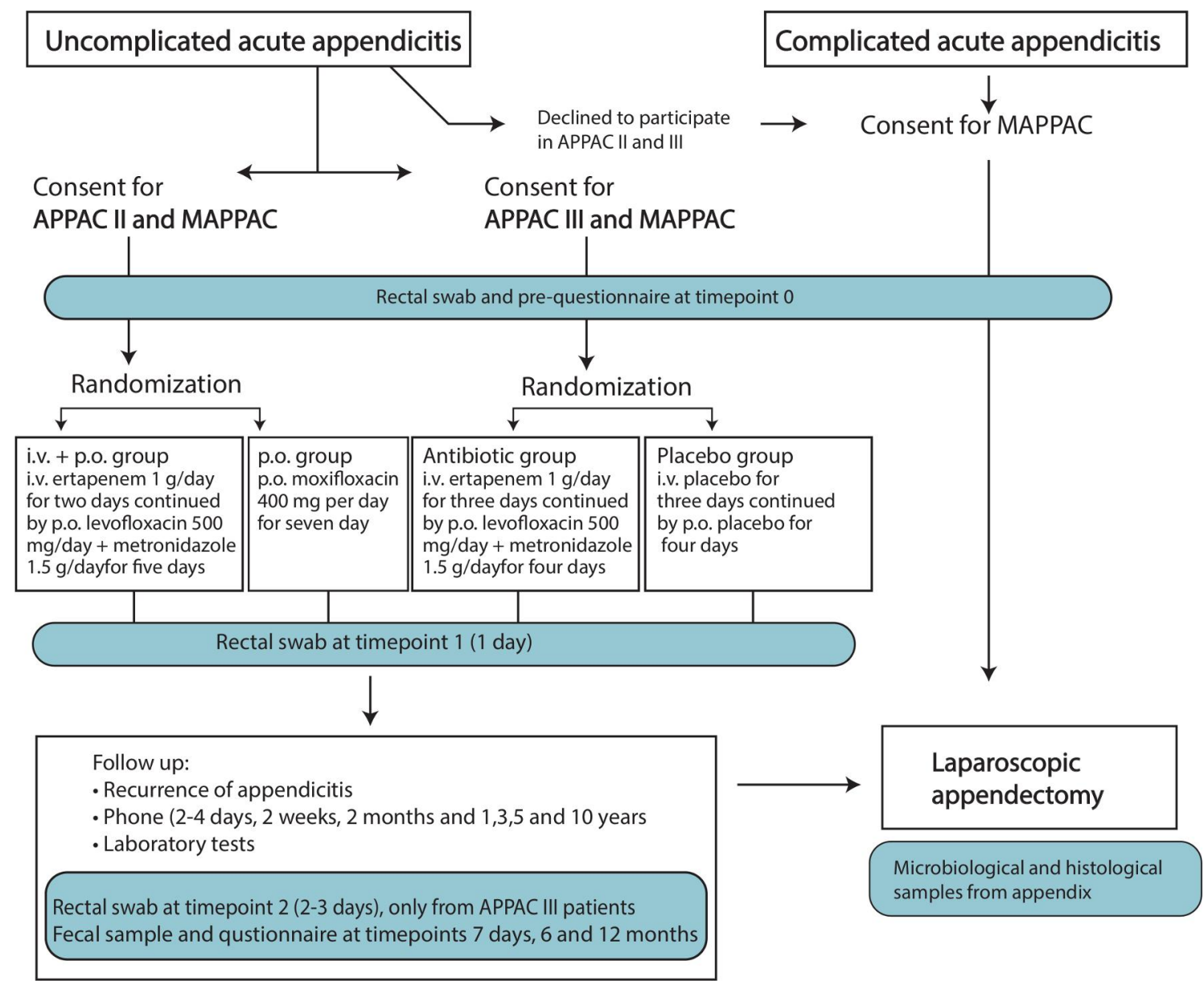

Figure 2 Flow chart of the study protocol. APPAC II, APPendicitis ACuta II; i.v, intravenous; MAPPAC, Microbiology APPendicitis ACuta; p.o., per oral.

Kuopio). In addition, all of the APPAC II and III trial patients having to undergo appendectomy either for a treatment failure during the primary hospitalisation or for suspected recurrence after a successful initial non-operative treatment will be enrolled in the MAPPAC trial at all 10 study hospitals (all 5 university and 5 central hospitals in Finland: Jyväskylä, Pori, Mikkeli, Seinäjoki and Rovaniemi).

\section{Study setting and feasibility}

The study was initiated at Turku University Hospital in April 2017, with the study commencing at all study centres by fall 2017. The majority of MAPPAC patient enrolment is evaluated to be completed in conjunction with the APPAC II and III trials latest by fall 2020. Patient recruitment in the MAPPAC trial will continue for a minimum of 5 years after APPAC II and III trial enrolment completion based on the planned microbiological assessment of late appendicitis recurrence.

\section{Interventional groups}

Four interventional groups within MAPPAC are defined as follows:

1. Patients with uncomplicated acute appendicitis participating in the APPAC II trial at Turku University Hospital (MAPPAC single-centre treatment arm) receiving either intravenous antibiotic therapy followed by p.o. antibiotics (intravenous+p.o.) or patients receiving p.o. antibiotic monotherapy (p.o.). Intravenous+p.o. group receives intravenous ertapenem $1 \mathrm{~g}$ once daily for 2 days followed by p.o. levofloxacin $500 \mathrm{mg}$ once daily and metronidazole $500 \mathrm{mg}$ three times per day for 5 days. P.o. group receives p.o. moxifloxacin $400 \mathrm{mg}$ once daily for 7 days.

2. Patients with uncomplicated acute appendicitis participating in the double-blinded APPAC III trial at all five university hospitals (MAPPAC multicentre treatment arm) receiving either antibiotic therapy or placebo treatment. Antibiotic treatment is intravenous ertapenem $1 \mathrm{~g}$ once per day for 3 days followed by p.o. levofloxacin $500 \mathrm{mg}$ once per day and metronidazole $500 \mathrm{mg}$ three times per day for 4 days. Placebo treatment entails intravenous placebo once per day for 3 days followed by p.o. placebo three times per day for 4 days.

3. Patients with uncomplicated acute appendicitis or suspected recurrent appendicitis undergoing appendectomy.

The patients in this group will undergo laparoscopic appendectomy either after declining to participate in the APPAC II or APPAC III trials (MAPPAC single-centre treatment arm at Turku University Hospital) or 
after suspected treatment failure of non-operative treatment or after presenting with suspected appendicitis recurrence after initial successful non-operative treatment (MAPPAC multicentre treatment arm, all APPAC II and III hospitals)

4. Patients with complicated acute appendicitis undergoing appendectomy. The patients in this group will undergo laparoscopic appendectomy and are eligible for enrolment only in the MAPPAC single-centre study arm at Turku University Hospital.

\section{Sample collection}

Rectal swabs are collected from all patients in the emergency department prior to antibiotic treatment or surgery (time point 0 ). Further, rectal swabs are collected on the first day for APPAC II patients and for APPAC III patients on the first day (Turku University Hospital) and on the third day (all five university hospitals). At each time point, two rectal swabs are collected: Amies-Coal transport swab for culturing methods (Sarstedt, Nümbrecht, Germany) and Puritan DNA/RNA swab (Puritan Medical products, Guilford, Maine) with shield fluid (Zymo Research, Irvine, Canada) for microbial DNA extraction. The shield fluid in this collection tube allows the transportation at room temperature.

Samples from the appendix are collected from the patients undergoing appendectomy for complicated or uncomplicated acute appendicitis and from the patients with suspected disease progression during the primary hospitalisation or appendicitis recurrence after successful initial non-operative therapy. Samples include routine histology as well as specific trial swabs and biopsies. The appendix is opened vertically with a sterile scalpel and two swabs are taken similar to the rectal swab (one for culture and one for DNA extraction). Three biopsies are taken at a distance of approximately $2 \mathrm{~cm}$ from the base of the appendix. All biopsies are stored at $-75^{\circ} \mathrm{C}$, one immediately and the rest two are first embedded in RNAlater solution (Thermo Fisher Scientific, Waltham, Massachusetts, USA). Possible appendicoliths are collected into a sterile container, frozen and stored at $-75^{\circ} \mathrm{C}$. If appendectomy is performed during office hours, appendiceal samples are collected by study personnel and an additional swab for anaerobic culture is then collected and cultured in connection with collection and immediately transferred into an anaerobic jar. During on call hours, the samples are collected by surgeons and the $-75^{\circ} \mathrm{C}$ appendiceal biopsy may not be collected. These appendiceal samples are collected only in the Turku University Hospital and at the other study hospitals only a swab sample (transport tube with DNA shield fluid) from the appendix of non-responders and patients with appendicitis recurrence is collected.

Additional serum samples are collected from all the patients recruited in MAPPAC trial at Turku University Hospital and for APPAC III trial at all five study hospitals for the identification of possible disease form specific inflammatory biomarkers in serum. The serum samples are divided into six aliquots prior freezing at $-75^{\circ} \mathrm{C}$.

\section{Questionnaire}

MAPPAC patients are asked to fill a primary questionnaire during the initial hospitalisation covering topics possibly affecting their GM profile: chronic diseases, special diets, smoking and alcohol consumption, travel history, antibiotic intake, other medications (12 months prior the sampling), consumption of probiotics and other dietary supplements, recent diarrhoea and/or vomiting, Bristol stool form scale estimate $^{35}$ measuring stool consistency (1 for very hard stool to 7 for diarrhoea) and the average number of defecations per day.

\section{Follow-up during the hospitalisation}

During the hospitalisation, the following parameters will be recorded every 24hours: pain assessed by Visual Analogue Scale (VAS), leucocyte count, C reactive protein (CRP), temperature and clinical findings at patient reassessment. If the patient is suspected of not responding to the randomised therapy based on clinical deterioration signs combined with laboratory findings (signs of peritonitis, persisting fever, increasing pain, white cell count or CRP), the patient will be operated based on the surgeon's decision and the reasons for proceeding to appendectomy will be recorded. For appendectomy, laparoscopic approach is recommended. The operative findings and the histopathology of the appendix will be recorded. After the initial hospitalisation, recurrent acute appendicitis will be diagnosed on a clinical basis and patients with a suspected recurrence of appendicitis will undergo a laparoscopic appendectomy.

\section{Follow-up}

The specific MAPPAC trial follow-up is conducted for patients also participating in the APPAC II and III trials with collection of three faecal samples at home (at 1 week, 6 months and 1year). Follow-up samples at home are not collected in MAPPAC interventional groups undergoing appendectomy. The follow-up faecal sample is collected into $10 \mathrm{~mL}$ DNA/RNA Shield Faecal Collection Tube (Zymo Research) enabling transportation and storage at room temperature prior to DNA extraction. Patients also deliver a faecal sample into a gel tube for culture purposes. A follow-up questionnaire is collected at 6 and 12 months consisting of the same questions as the preliminary questionnaire covering the time between hospital discharge and the follow-up time point. Other follow-up for patients in APPAC II trial will include a phone interview at 1 week after discharge, APPAC III trial patients have their first follow-up call 2-4 days after discharge from the hospital and then at 10 days, both trials at 2 months and at 1, 3, 5 and 10 years. The follow-up for APPAC III patients will include laboratory tests (leucocyte count, CRP and additional follow-up serum samples).

\section{Outcome parameters}

Based on the MAPPAC trial design, no specific primary endpoint can be determined. The following parameters will be recorded for all patients: age, gender, body mass index, 
clinical findings on admission (tympanic temperature, nausea, pain or tenderness in the right lower abdominal quadrant, pain migration, VAS pain scores, white cell count, CRP, findings on CT imaging) and data on primary and follow-up questionnaires. Blood cultures will be obtained from patients with complicated acute appendicitis and for APPAC II and III trial patients at Turku University Hospital.

The following outcome parameters will be assessed based on the sample types collected:

\section{Parameters from the appendix, that is, patients undergoing appendectomy}

Operative details and histopathology of the appendix, host transcriptomics, proteomics and immunohistochemical analysis from appendiceal biopsy, possible appendicolith composition, morphology and classification.

\section{Microbiological parameters from the rectal and appendiceal swabs, faecal and appendiceal samples}

Microbial profile, metagenome and metatranscriptome, name of different identified bacterial species, number of species identified both by NGS and culture methods, antimicrobial susceptibility test results, the presence of AMR-related genes by molecular analysis methods, bacterial genome data of AMR or virulence genes, all microbial data combined with clinical and additional data.

\section{Serum samples}

In order to compare possible differences between patients with successful antibiotic therapy to patients with either failed antibiotic therapy or complicated acute appendicitis, the serum samples will be analysed to identify possible inflammatory or immunological markers. Different cytokines, chemokines and growth factors as well as serum metabolome will be included in these analyses. Additional analysis include the level and activity of CD73 and soluble vascular adhesion protein-1. List for analytes that will be screened is provided in the online supplementary methods.

Laboratory methods used in the trial to analyse the collected samples are described in detail in the online supplementary material.

\section{Statistical analysis}

Based on the explorative research nature of the MAPPAC study, there is not enough information available about the study aims to enable sample size calculations. Categorical variables of the study will be characterised using frequencies and percentages. For continuous variables means and $\mathrm{SD}$ or medians with range and 25th and 75th percentiles will be used. In case of categorical outcomes, groups will be compared using Pearson's $\chi^{2}$ test and if further analyses will be needed, logistic regression models will be used. Group differences in continuous variables will be evaluated using independent samples t-test, analysis of variance or non-parametric methods, if needed. Associations between continuous variables will be evaluated using correlation coefficients and linear regression analysis and if adjustments are needed, linear models will be used. Continuous outcomes measured in several time points will be analysed using linear mixed models. For categorical outcomes with repeated measurements, generalised linear mixed models will be used. The assumptions of the methods will be checked for justification of the analyses and transformations will be used for the variables, if needed. The study site differences will be evaluated in statistical models and if major differences are detected, more complicated statistical models will be used in the analyses. Two-sided $p$ values will be used and $p$ values less than 0.05 will be considered statistically significant. The measurements with missing data will automatically be excluded from the analyses of the variables in concern. Statistical analyses will be performed using SAS System for Windows, V.9.4 and JMP Pro V.13 or later versions (SAS Institute).

\section{Patients and public involvement}

The MAPPAC research questions and outcome measures were based on the results of original APPAC trial ${ }^{25}$ and the study protocol was developed together with the study group surgeons, clinical microbiologists and immunologists. Patients were not directly involved in the design of this study and the burden of study participation was not assessed by patients themselves. On recruitment, patients are well informed of all aspects of the trial including antibiotic or symptomatic therapy of CT-confirmed uncomplicated acute appendicitis, difference between complicated and uncomplicated acute appendicitis, treatment success, possible late recurrence and safety in order to help patients make an informed decision about trial participation. Patients also receive additional instructions in a phone call made prior to follow-up sample collection at 6 and 12 months. After completion of data collection and analysis, the patients will be informed of the study results and they will be provided with an opportunity to ask further questions.

\section{DISSEMINATION}

\section{Data collection and confidentiality}

All data and samples are handled confidentially and the information in the datasets is non-identifiable. Data are gathered during the emergency room visit, hospitalisation for acute appendicitis, clinical observations and follow-up phone calls. The main investigators will be in charge of the common database with full access to the data which is, otherwise strictly limited. As the MAPPAC and APPAC II and III trials are based on the same patient population, the interventions partly overlap and the enrolled patients are informed about this overlapping of the trials and the acquired data.

\section{Withdrawal}

Patients are informed of their right to withdraw from the study without explanation at any time. In case of patient withdrawal, they will be asked for permission to use their data.

\section{Dissemination plan}

Results from this trial and reported in articles which will be published in peer-reviewed journals. Results are also 
presented at national and international conferences to further distribute this research.

\section{DISCUSSION}

As non-operative treatment for uncomplicated acute appendicitis has been shown to be efficient and safe also at long term ${ }^{25-28} 3136$ and cost-effective, ${ }^{37}$ understanding both the aetiology of the different appendicitis forms and potentially predicting the recurrences has become of utmost clinical importance in order to thoroughly evaluate all the optimisation of the different treatment options. The MAPPAC trial aims to assess this largely unknown microbiological aetiology of the different disease forms of acute appendicitis. Further, the whole microbial entity, that is, profile both in the appendix and in gut is studied, and their role in the disease severity and form is assessed. In addition, the effects of antibiotic and placebo treatments on the GM profile and the effects of the duration of the hospital stay on the AMR reservoir of the GM will be assessed. With the longitudinal study design, both the immediate and long-term effects of the antibiotic treatment on GM can be analysed. As the hospital stay differs in APPAC II and III patients, the effects of the duration of the hospital stay on the emergence of resistant bacteria and AMR reservoir can be evaluated. Moreover, the comparison of the effects of antibiotic treatment on GM between two different administration routes (p.o. and intravenous+p.o.) is possible.

In the 5-year follow-up of the original APPAC trial, 39\% $(n=100)$ of the antibiotic group patients underwent appendectomy for either during the primary hospitalisation or for suspected late appendicitis recurrence. Out of these 100 patients, 85 were operated on for suspected recurrence and 78 patients had a histopathologically confirmed acute appendicitis. ${ }^{26}$ Understanding the pathophysiology and contributing factors in recurrent appendicitis are of vital clinical importance in evaluating the optimal treatment paradigms for uncomplicated acute appendicitis. Therefore, MAPPAC trial is assessing both initial non-responders to antibiotic therapy and recurrent appendicitis after successful initial antibiotic treatment and these results may provide novel tools to predict the potential recurrence risk and thus help to assess the optimal treatment choice for patients with uncomplicated acute appendicitis.

\section{Strengths and limitations}

MAPPAC has a strong synergy between APPAC II and III studies enabling the enrolment of both uncomplicated and complicated acute appendicitis patients in a large prospective series with associated clinical data to be assessed in conjunction with the microbiological and immunological findings. In many aspects, MAPPAC is an exploratory study of possible associations of whole microbial community and host immune characteristics with uncomplicated versus complicated appendicitis and antibiotic response among patients in clinical trials treated with and without antibiotics. MAPPAC trial aims to generate hypotheses to better understand the role of disease progression and host susceptibility for future studies, that is determination of one primary outcome is insufficient for the study, as several factors are indispensable for the understanding of aetiology and the effects of antibiotics on GM all provided with this unique set of microbiological samples. To our knowledge, only one previous study ${ }^{12}$ has characterised the adult appendix microbiota during appendicitis in adult patients using NGS technique and to date no reports on the structure and physicochemical contents of appendicoliths exists. Using these assessments is a strength in our study. Further, to our knowledge, this is the first trial aiming to prospectively assess the possible microbiological and/or immunological aetiology of appendicitis recurrence after a successful initial conservative treatment with antibiotics or symptomatic therapy and primary non-responders to conservative treatment of uncomplicated acute appendicitis. One of the main hypothesis of the MAPPAC study is that the microbial composition of appendix differs between CT differentiated complicated and uncomplicated appendicitis. Therefore, strong element of the study is that all patients included in the study are imaged with CT protocol. CT scan is the gold standard for acute appendicitis imaging and this near perfect accuracy of CT adds to the reliability of the clinical data and appendicitis severity also in the patients with uncomplicated acute appendicitis without a histopathological confirmation of the appendix.

The study limitations include the difficult challenge of conducting prospective clinical trials in the emergency setting. It is expected that all eligible patients may not be evaluated for enrolment or some patients may not have all study samples available as the recruitment is performed by a large number of surgeons on call. The lack of healthy control group is a limitation in the study regarding both the aetiology and in determining the effects of antibiotics on GM, as the results cannot be fully distinguished from the effects of acute appendicitis.

\section{Author affiliations}

${ }^{1}$ Institute of Biomedicine, Research Center for Cancer, Infections and Immunity, University of Turku, Turku, Finland

${ }^{2}$ Division of Digestive Surgery and Urology, Turku University Hospital, Turku, Finland ${ }^{3}$ Microbiome Biobank, Faculty of Medicine, University of Turku, Turku, Finland ${ }^{4}$ Department of Clinical Microbiology, Turku University Hospital, Turku, Finland ${ }^{5}$ Department of Surgery, University of Turku, Turku, Finland

${ }^{6}$ Medicity and Institute of Biomedicine, University of Turku, Turku, Finland ${ }^{7}$ Department of Hospital Hygiene and Infection Control, Turku University Hospital, Turku, Finland

${ }^{8}$ Department of Biostatistics, University of Turku, Turku, Finland

${ }^{9}$ Satakunta Hospital District, Pori, Finland

Collaborators Due to the multicentre nature of the trial, not all supporting researchers are mentioned by name in the protocol article. In addition, The APPAC collaborative study group lead by primary investigator Paulina Salminen includes the following contributors: Sallinen V., Leppäniemi A., Rautio T., Meriläinen S., Nordström P., Laukkarinen J., Rantala T., Savolainen H., Aarnio M., Mattila A., Haijanen J., Sävelä E-L., Imre I., Paajanen H., Rintala J., Pinta T., Sippola T., and Böckerman P. Local investigators, who are responsible for execution of the APPAC II and/or APPAC III trials in addition to execution of the applicable parts of the MAPPAC trial and valid data gathering. The surgical departments of the following Finnish Hospitals contribute to the execution of this trial: University hospitals of Turku, Helsinki, Oulu, Tampere, and Kuopio, central hospitals of Jyväskylä, Pori, Mikkeli, Seinäjoki, and Rovaniemi. In addition, we acknowledge all supporting surgeons, 
radiologists, emergency medicine physicians, nurses and technical staff in the laboratory.

Contributors All authors have contributed to the design of this trial protocol. PS is the chief investigator. PS, SV, AJH, EM, SJ, JG and SS have initiated the project. The protocol was drafted by PS, which was refined by SV, AJH, EM, SS, SJ, JG, HM, EE and SH. Statistical advice was provided by SH. SV was responsible for drafting the manuscript, which was refined by PS, AJH and EM. All authors read and approved the final manuscript.

Funding This work was supported by clinical research grants from Sigrid Juselius foundation, the Academy of Finland, Mary and Georg C. Ehrnrooth foundation, The Maud Kuistila Memorial Foundation, Doctoral Programme of Clinical Investigation of University of Turku, Tyks-Sapa Research Funds, Finland, Turku University and Turku University foundation. This is a researcher-oriented trial.

Competing interests None declared.

Patient consent for publication Not required.

Ethics approval This protocol has been accepted by the Ethical Committee of the Hospital districtof Southwest Finland (Turku University Hospital) and Finnish Medicines Agency (Fimea). The trial will be conducted in compliance with the Declaration of Helsinki.

Provenance and peer review Not commissioned; externally peer reviewed.

Open access This is an open access article distributed in accordance with the Creative Commons Attribution Non Commercial (CC BY-NC 4.0) license, which permits others to distribute, remix, adapt, build upon this work non-commercially, and license their derivative works on different terms, provided the original work is properly cited, appropriate credit is given, any changes made indicated, and the use is non-commercial. See: http://creativecommons.org/licenses/by-nc/4.0/.

\section{REFERENCES}

1. Addiss DG, Shaffer N, Fowler BS, et al. The epidemiology of appendicitis and appendectomy in the United States. Am J Epidemiol 1990;132:910-25.

2. Ferris M, Quan S, Kaplan BS, et al. The global incidence of appendicitis: a systematic review of population-based studies. Ann Surg 2017;266:237-41.

3. Livingston EH, Fomby TB, Woodward WA, et al. Epidemiological similarities between appendicitis and diverticulitis suggesting a common underlying pathogenesis. Arch Surg 2011;146:308-14.

4. Forbes GB, Lloyd-Davies RW. Calculous disease of the vermiform appendix. Gut 1966;7:583-92.

5. Lamps LW. Infectious causes of appendicitis. Infect Dis Clin North Am 2010;24:995-1018.

6. Swidsinski A, Dörffel Y, Loening-Baucke V, et al. Acute appendicitis is characterised by local invasion with Fusobacterium nucleatum/ necrophorum. Gut 2011;60:34-40.

7. Zhong D, Brower-Sinning R, Firek B, et al. Acute appendicitis in children is associated with an abundance of bacteria from the phylum Fusobacteria. J Pediatr Surg 2014;49:441-6.

8. Jindal N, Kaur GD, Arora S, et al. Bacteriology of acute appendicitis with special reference to anaerobes. Indian J Pathol Microbiol 1994;37:299-305.

9. Martirosian G, Bulanda M, Wójcik-Stojek B, et al. Acute appendicitis: the role of enterotoxigenic strains of Bacteroides fragilis and Clostridium difficile. Med Sci Monit 2001;7:382-6.

10. Chen $\mathrm{C}-\mathrm{Y}$, Chen $\mathrm{Y}-\mathrm{C}, \mathrm{Pu} \mathrm{H}-\mathrm{N}$, et al. Bacteriology of acute appendicitis and its implication for the use of prophylactic antibiotics. Surg Infect 2012;13:383-90.

11. Reinisch A, Malkomes $P$, Habbe N, et al. Bad bacteria in acute appendicitis: rare but relevant. Int J Colorectal Dis 2017;32:1303-11.

12. Guinane CM, Tadrous A, Fouhy F, et al. Microbial composition of human appendices from patients following appendectomy. MBio 2013;4:12.

13. Peeters T, Penders J, Smeekens SP, et al. The fecal and mucosal microbiome in acute appendicitis patients: an observational study. Future Microbiol 2019;14:111-27.

14. Bennion RS, Baron EJ, Thompson JE, et al. The bacteriology of gangrenous and perforated appendicitis--revisited. Ann Surg 1990;211:165-71.
15. Rivera-Chavez FA, Peters-Hybki DL, Barber RC, et al. Innate immunity genes influence the severity of acute appendicitis. Ann Surg 2004;240:269-77.

16. Dethlefsen L, Relman DA. Incomplete recovery and individualized responses of the human distal gut microbiota to repeated antibiotic perturbation. Proc Natl Acad Sci U S A 2011;108 Suppl 1:4554-61.

17. Jernberg C, Löfmark S, Edlund C, et al. Long-Term ecological impacts of antibiotic administration on the human intestinal microbiota. Isme J 2007;1:56-66.

18. Manichanh C, Rigottier-Gois L, Bonnaud E, et al. Reduced diversity of faecal microbiota in Crohn's disease revealed by a metagenomic approach. Gut 2006;55:205-11.

19. Qin J, Li Y, Cai Z, et al. A metagenome-wide association study of gut microbiota in type 2 diabetes. Nature 2012;490:55-60.

20. Lindgren M, Löfmark S, Edlund C, et al. Prolonged impact of a oneweek course of clindamycin on Enterococcus spp. in human normal microbiota. Scand J Infect Dis 2009;41:215-9.

21. Raymond F, Ouameur AA, Déraspe M, et al. The initial state of the human gut microbiome determines its reshaping by antibiotics. Isme J 2016;10:707-20.

22. Jakobsson HE, Jernberg $C$, Andersson AF, et al. Short-Term antibiotic treatment has differing long-term impacts on the human throat and gut microbiome. PLoS One 2010;5:e9836.

23. Buelow E, Bello González TDJ, Fuentes S, et al. Comparative gut microbiota and resistome profiling of intensive care patients receiving selective digestive tract decontamination and healthy subjects. Microbiome 2017;5.

24. Card RM, Mafura M, Hunt T, et al. Impact of ciprofloxacin and clindamycin administration on gram-negative bacteria isolated from healthy volunteers and characterization of the resistance genes they harbor. Antimicrob Agents Chemother 2015;59:4410-6.

25. Salminen P, Paajanen H, Rautio T, et al. Antibiotic therapy vs appendectomy for treatment of uncomplicated acute appendicitis: the APPAC randomized clinical trial. JAMA 2015;313:2340-8.

26. Salminen P, Tuominen R, Paajanen $\mathrm{H}$, et al. Five-Year follow-up of antibiotic therapy for uncomplicated acute appendicitis in the APPAC randomized clinical trial. JAMA 2018;320:1259-65.

27. Sakran JV, Mylonas KS, Gryparis A, et al. Operation versus antibiotics--The "appendicitis conundrum" continues: A metaanalysis. J Trauma Acute Care Surg 2017;82:1129-37.

28. Sallinen V, Akl EA, You JJ, et al. Meta-analysis of antibiotics versus appendicectomy for non-perforated acute appendicitis. Br J Surg 2016;103:656-67.

29. Podda M, Gerardi C, Cillara N, et al. Antibiotic treatment and appendectomy for uncomplicated acute appendicitis in adults and children: a systematic review and meta-analysis. Ann Surg 2019. doi:10.1097/SLA.0000000000003225. [Epub ahead of print: 31 Jan 2019].

30. Harnoss JC, Probst P, Büchler MW, et al. Antibiotics versus appendicectomy for the treatment of uncomplicated acute appendicitis: an updated meta-analysis of randomised controlled trials by Rollins et al. World J Surg 2017;41:2411.

31. Rollins KE, Varadhan KK, Neal KR, et al. Antibiotics versus appendicectomy for the treatment of uncomplicated acute appendicitis: an updated meta-analysis of randomised controlled trials. World J Surg 2016;40:2305-18.

32. Chan A-W, Tetzlaff JM, Altman DG, et al. SPIRIT 2013 statement: defining standard protocol items for clinical trials. Ann Intern Med 2013;158:200-7.

33. Haijanen J, Sippola S, Grönroos J, et al. Optimising the antibiotic treatment of uncomplicated acute appendicitis: a protocol for a multicentre randomised clinical trial (APPAC II trial). BMC Surg 2018;18:117.

34. Sippola S, Grönroos J, Sallinen V, et al. A randomised placebocontrolled double-blind multicentre trial comparing antibiotic therapy with placebo in the treatment of uncomplicated acute appendicitis: APPAC III trial study protocol. BMJ Open 2018;8:023623.

35. Lewis SJ, Heaton KW. Stool form scale as a useful guide to intestinal transit time. Scand J Gastroenterol 1997;32:920-4.

36. Vons C, Barry C, Maitre S, et al. Amoxicillin plus clavulanic acid versus appendicectomy for treatment of acute uncomplicated appendicitis: an open-label, non-inferiority, randomised controlled trial. The Lancet 2011;377:1573-9.

37. Sippola S, Grönroos J, Tuominen R, et al. Economic evaluation of antibiotic therapy versus appendicectomy for the treatment of uncomplicated acute appendicitis from the APPAC randomized clinical trial. Br J Surg 2017;104:1355-61. 